\title{
VERIFICATION TEST \\ PROBLEMS
}

B. Moran

August 10, 2007

15th APS topical conference on Shock Compression of Condensed Matter Kohala Coast, HI, United States June 24, 2007 through June 29, 2007 
This document was prepared as an account of work sponsored by an agency of the United States Government. Neither the United States Government nor the University of California nor any of their employees, makes any warranty, express or implied, or assumes any legal liability or responsibility for the accuracy, completeness, or usefulness of any information, apparatus, product, or process disclosed, or represents that its use would not infringe privately owned rights. Reference herein to any specific commercial product, process, or service by trade name, trademark, manufacturer, or otherwise, does not necessarily constitute or imply its endorsement, recommendation, or favoring by the United States Government or the University of California. The views and opinions of authors expressed herein do not necessarily state or reflect those of the United States Government or the University of California, and shall not be used for advertising or product endorsement purposes.

This work was performed under the auspices of the U. S. Department of Energy by University of California, Lawrence Livermore National Laboratory under Contract W-7405-Eng-48. 


\title{
VERIFICATION TEST PROBLEMS
}

\author{
B. Moran \\ Lawrence Livermore National Laboratory, Livermore CA 94551
}

\begin{abstract}
We present analytic solutions to two test problems that can be used to check the hydrodynamic implementation in computer codes designed to calculate the propagation of shocks in spherically convergent geometry. Our analysis is restricted to fluid materials with constant bulk modulus. In the first problem we present the exact initial acceleration and pressure gradient at the outer surface of a sphere subjected to an exponentially decaying pressure of the form $P(t)=P_{0} e^{-\alpha t}$. We show that finely-zoned hydro-code simulations are in good agreement with our analytic solution. In the second problem we discuss the implosions of incompressible spherical fluid shells and we present the radial pressure profile across the shell thickness. We also discuss a semi-analytic solution to the timeevolution of a nearly spherical shell with arbitrary but small initial 3-dimensional (3-D) perturbations on its inner and outer surfaces.
\end{abstract}

Keywords: Convergent geometry, spherical implosion, compressible fluid, code verification.

PACS: 02.70-c, 46.15-x, 47.40.-x, 83.10Ff.

\section{INTRODUCTION}

In the first problem we consider a fluid sphere subjected to an exponentially decaying pressure of the form $P(t)=P_{0} e^{-\alpha t}$ where $t$ is the time and $\alpha$ is the decay constant. Although Guderley [1] has previously addressed the problem of strong shocks in a convergent geometry, his work dealt only with an ideal gas. Extensions of Guderley's work were made by Ponchaut et al [2] who considered a series expansion of the solution and by $\mathrm{Wu}$ and Roberts [3] who explored the solution for a van der Waals gas. Here we consider a material with a constant bulk modulus and include the added complication of a prescribed pressure on an outer boundary. In Guderley's solved problem, there is no applied pressure at a given radius and thus no distance scale and his solution is self-similar whereas ours is not.

In the second problem, we consider a spherical shell subjected to a weak shock (peak pressure, $P_{0}$, much less than the bulk modulus $K$ of the shell). Sharp [4], Blake [5], and Larson [6] worked out the solution of the wave motion produced when a pressure is applied to the interior surface of a spherical cavity in an ideally elastic medium. Their interest was in geophysical applications and seismic decoupling. More recently, Moran [7] adapted this earlier work by considering a convergent instead of a divergent spherical wave and Williams et al [8] used eigenfunction techniques to address this problem. Here we focus on the implosion of that shell and its subsequent large deformation.

\section{INITIAL PRESSURE GRADIENT AND OUTER ACCELERATION}

Consider a fluid sphere of radius $a$ that is subjected to a time dependent pressure. This fluid is such that the pressure, $P$, is linearly related to the compression, $\mu$, through $P=K \mu$ where $K$ is a 
constant and $\mu$ is defined as $\rho / \rho_{0}-1$ where $\rho$ is the density and $\rho_{0}$ is the initial uncompressed density. The initial pressure gradient $d P / d R$ and the initial acceleration at the surface of the sphere may be obtained by considering energy conservation in a small element at the surface. We first consider the case where the pressure increases as $P(t)=P_{0} e^{\beta t}$, with $\beta>0$ chosen such that the initial pressure gradient is identically zero. We will compute $\beta$ and then use it to obtain the initial pressure gradient and acceleration at the surface for an arbitrary pressure of the form $P(t)=P_{0} e^{-\alpha t}$.

\section{Calculation of $\boldsymbol{\beta}$}

When the initial pressure gradient, $d P / d R$, is identically zero, the initial acceleration is also identically zero and the outer velocity $V_{0}$ is constant in time but has a spatial gradient $d V / d R$. In that case, the kinetic energy reduces to an integral of a polynomial in the spatial variable $R$. The term proportional to the first power of $t$ in the evaluated integral is independent of $\beta$ and is given by $2 \pi a^{2} P_{0} V_{0}$ where $V_{0}$ is the initial material speed at the surface. The second time derivative of the kinetic energy evaluated at $t=0$ is given by:

$$
\begin{aligned}
& \left.\frac{d^{2}}{d t^{2}} K E(t)\right|_{t=0}=\pi \rho_{0} a^{2} V_{0}^{2} U_{s 0} \\
& {\left[\beta \frac{P_{0}\left(5 K+P_{0}\right)}{K\left(K+P_{0}\right)}-4 \frac{U_{s 0}\left(K+2 P_{0}\right)}{a\left(K+P_{0}\right)}+\left.4 \frac{K}{P_{0}} \frac{d V}{d R}\right|_{t=0}\right]}
\end{aligned}
$$

Where $V_{0}=P_{0} /\left(\rho_{0} U_{s 0}\right), U_{s 0}$ the initial shock velocity is $\left[\left(K+P_{0}\right) / \rho_{0}\right]^{1 / 2}$, and the initial velocity gradient $d V / d R$ is $\frac{\beta P_{0}\left(2 K+P_{0}\right)}{2 U_{s 0}^{3} \rho_{0}^{2}\left(U_{s 0}-V_{0}\right)}$. The internal energy is composed of two parts: a part $E_{s}(t)$ that comes from the energy of shocking the material and a part $E_{P d \nu}(t)$ that comes from the isentropic expansion or compression following the initial shock. See Fig 1. By equating the product of the applied force and the velocity with the rate of change of the total energy and expanding to first order in time, we obtain an algebraic expression for $\beta$. Solving, we get:

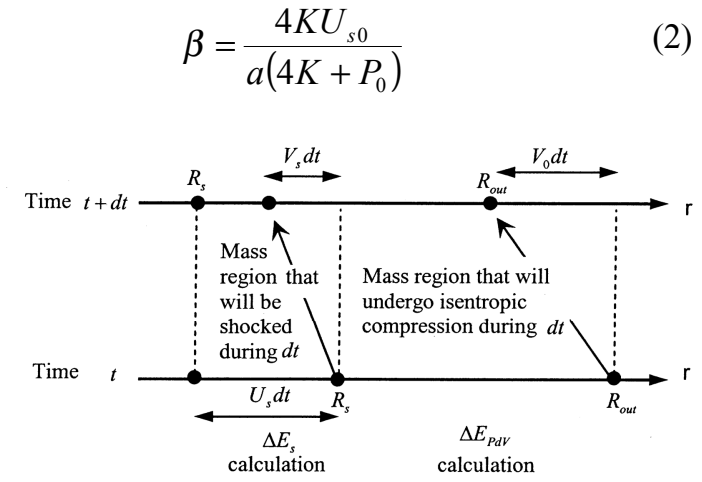

Figure 1. Schematic showing the mass region that will be shocked (for $\Delta E_{s}$ calculation) and the mass region that will undergo isentropic compression (for $\Delta E_{P d V}$ calculation) during $d t$. The solid oblique arrows indicate the motion of material particles that were initially located at the outer radius $R_{\text {out }}$ and the shock front radius $R_{s} . V_{0}$ is the speed of a particle initially at $R_{\text {out }}$.

\section{Decaying outer pressure}

When the initial pressure gradient is not zero, hydrodynamic attenuation [9] must be accounted for. Hydrodynamic attenuation reduces the pressure gradient from what it would have been without it by the factor $\left(U_{s 0}-V_{0}\right) /\left(U_{s 0}-V_{0}+f U_{s 0}\right)$, Where $f$ is given by $2 P_{0} K /\left[\left(P_{0}+K\right)\left(P_{0}+4 K\right)\right]$. We now return to our original problem in which the pressure decays exponentially as $P(t)=P_{0} e^{-\alpha t}$ and note that at the initial time and without hydrodynamic attenuation we have:

$$
\begin{aligned}
& \left.\frac{d P}{d R}\right|_{t=0, \text { NoAtten }}=\lim _{\Delta R \rightarrow 0} \frac{P_{0} e^{-\alpha t}-P_{0} e^{\beta t}}{\Delta R}=\lim _{\Delta t \rightarrow 0} \frac{P_{0} e^{-\alpha \Delta t}-P_{0} e^{\beta \Delta t}}{\left(U_{s 0}-V_{0}\right) \Delta t} \\
& =-\frac{P_{0}(\alpha+\beta)}{\left(U_{s 0}-V_{0}\right)}
\end{aligned}
$$

Thus the exact initial pressure gradient is

$$
\begin{gathered}
\left.\frac{d P}{d R}\right|_{t=0, \text { exact }}=-\frac{P_{0}\left(\alpha+\frac{4 K U_{s 0}}{a\left(4 K+P_{0}\right)}\right)}{\left(U_{s 0}-V_{0}+f U_{s 0}\right)} \\
=-\frac{P_{0}\left(\alpha P_{0} a+4 K\left(U_{s 0}+\alpha a\right)\right)\left(P_{0}+K\right)}{U_{s 0} a\left(4 K+P_{0}\right)\left[K+f\left(P_{0}+K\right)\right]}
\end{gathered}
$$


And the exact initial acceleration is:

$$
\begin{aligned}
& \left.\frac{d^{2} R_{\text {out }}}{d t^{2}}\right|_{t=0}=-\frac{d P /\left.d R\right|_{t=0}}{\rho_{0}\left(1+P_{0} / K\right)} \\
& =\frac{P_{0}\left(\alpha P_{0} a+4 K\left(U_{s 0}+\alpha a\right)\right)}{\left(4 K+3 P_{0}\right) U_{s 0} \rho_{0} a}
\end{aligned}
$$

There are two special cases of interest: first, in the limit of a very weak shock $\left(P_{0}<<K\right)$, the initial acceleration becomes:

$$
\left.\frac{d^{2} R_{\text {out }}}{d t^{2}}\right|_{t=0, \text { VeryWeakShock }}=\frac{P_{0}}{a \rho_{0}}\left(1+\frac{a \alpha}{U_{s 0}}\right)
$$

And second, for the time-independent pressure (i.e. $\alpha=0$ ), the initial acceleration reduces to:

$$
\left.\frac{d^{2} R_{\text {out }}}{d t^{2}}\right|_{t=0, \alpha=0}=\frac{4 K P_{0}}{\left(4 K+3 P_{0}\right) \rho_{0} a}
$$

Which in the limit of a very strong shock $\left(P_{0}\right.$ $>>K$ ) becomes:

$$
\left.\frac{d^{2} R_{\text {out }}}{d t^{2}}\right|_{t=0, \alpha=0, K / P_{0} \rightarrow 0}=\frac{4 K}{3 \rho_{0} a}
$$

Only in the case of a time-independent pressure, can the initial acceleration be expressed in a nondimensional form as a function of the shock strength parameter $x=P_{0} / K$ as

$$
\left.\frac{d^{2} R_{\text {out }}}{d t^{2}}\right|_{\text {Normalized }, t=0, \alpha=0}=\frac{4}{(4+3 x)}
$$

Where the normalized acceleration is the acceleration divided by $P_{0} / a \rho_{0}$. As defined in this paper, both $U_{s 0}$ and $V_{0}$ are positive however note that the shock and particle velocities point toward the origin for a compressive outer pressure.

We have verified that the CALE [10] hydrodynamic code in the 1-D configuration is consistent with Eq. 9. In Fig. 2, we show the results of our simulations along the analytic solution.

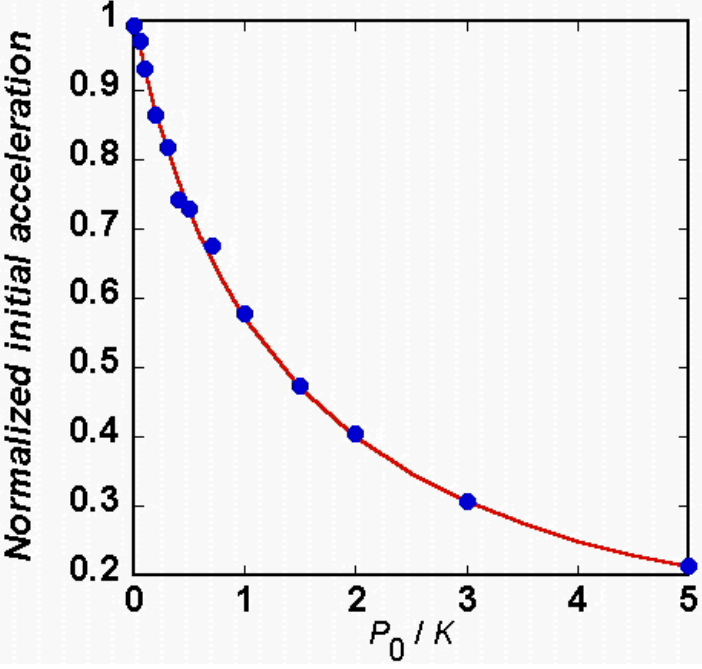

FIGURE 2. Initial acceleration normalized by $P_{0} / a \rho_{0}$. The symbols are from finely-zoned CALE [10] simulations and the line is from Eq. 9. The acceleration was calculated by taking the time-derivative of the velocity at the surface of the sphere. The noise in the data is due to the numerical differentiation and is on the order of the size of the symbols.

\section{IMPLOSION OF A SPHERICAL SHELL}

The implosion of an incompressible shell was discussed in Moran [7]. For the exponentially decaying pressure, the acceleration at the outer surface of the shell is given by the simple general expression:

$$
a_{o}(t)=\frac{\frac{-2 P_{0} e^{-\alpha t}}{\rho_{0}}+3 V_{o}^{2}(t)+r V_{o}(t)\left[r V_{i}(t)-4 V_{o}(t)\right]}{2 r(t)\left[R_{o}(t)-R_{i}(t)\right]}
$$

Where the subscript $o$ refers to values at the outer surface, subscript $i$ refers to values at the inner surface, $V(t)$ is the velocity, $R(t)$ is the radius, and $r(t)$ is the ratio $R_{o}(t) / R_{i}(t)$. Eq. (10) can be derived by equating the rate of change of kinetic energy of the shell $\frac{d}{d t} \int_{R_{i}(t)}^{R_{o}(t)} \frac{1}{2} 4 \pi \rho_{0} R^{2}[V(r)]^{2} d R$ with the rate of work done by the pressure applied to the outside surface $4 \pi\left[R_{o}(t)\right]^{2} P_{0} e^{-\alpha t} V_{o}(t)$. Conservation of volume implies that the particle velocity within the shell at location $R$ is 
proportional to $1 / R^{2}$ and the kinetic energy can be expressed in a closed form. Differentiation and the chain rule then yield Eq. (10). The two ODE's that need to be solved simultaneously are then $d V_{o}(t) / d t$ $=a_{o}(t)$ where $a_{o}$ is given by Eq. (10), and $d R_{o}(t) / d t$ $=V_{o}(t)$. The equivalent plastic strain across the shell at any location $R$ and time $t$ is given by:

$$
e_{p}=\frac{2}{3} \ln \left[1-\frac{R_{i}^{3}(t)-R_{i}^{3}(0)}{R^{3}(t)}\right.
$$

where $R_{i}(0)$ is the initial inner radius, and $\ln$ is the natural logarithm. The pressure at any location $R$ within the shell is obtained by equating the pressure gradient to the negative product of the density and the acceleration and then integrating. If there is no pressure applied to the inner surface of the shell, the pressure at any location $R$ within the shell is composed of three terms: a term inversely proportional to the fourth power of the radius, a term inversely proportional to the radius and a constant term. The pressure is given by:

$\rho_{0}\left(-\frac{R_{o}^{4} V_{o}^{2}}{2 R^{4}}+\frac{a_{o} R_{o}^{2}+2 R_{o} V_{o}^{2}}{R}+\frac{r^{4} V_{o}^{2}}{2}-a_{o} R_{o} r-2 V_{o}^{2} r\right)$

where $r$ is the ratio $R_{o} / R_{i}$ and $a_{o}$ is the acceleration at the outer surface given by Eq. 10. The location of the peak pressure and its magnitude are determined by differentiating the expression above with respect to $R$ and setting the derivative equal to zero. The plastic strain rate at any location $R\left(R_{i}<\right.$ $R<R_{o}$ ) is inversely proportional to the cube of $R$. It is also linearly proportional to the product of the instantaneous outer surface velocity of the shell $V_{o}$ and the square of the instantaneous outer radius $R_{o}$.

To simulate an incompressible fluid using an explicit finite difference code, we used a bulk modulus several orders of magnitude larger than the applied pressure. We found when simulating 3D perturbation growth during the implosion of thin shells -such as those used in laser fusion- that a minimum mesh resolution of about 30 zones per wavelength was required to qualitatively describe the amplitude phase-reversals resulting from the feed-through between the inner and outer boundaries of the shell. In Moran [7] we describe extensions to compressible fluids.

\section{DISCUSSION}

Extension of this work to cylindrical geometry is possible; it provides a way to check hydro-codes and compare the efficiency of various algorithms.

\section{ACKNOWLEDGEMENTS}

The author would like to acknowledge various discussions with Eric Nelson, Karnig Mikaelian, Hank Shay, and Yogi Gupta.

\section{REFERENCES}

1. Guderley, G. Starke kugelige und zylindrische Verdichtungstöße in der Nahe des Kugelmittelpunktes bzv. Der Zylinderische, Luftfahrtforschung 19, 302-312 1942.

2. Ponchaut, N. F., Honung, H. G., Pullin, D. I., and Mouton, C. A. "On imploding cylindrical and spherical shock waves in a perfect gas" J. Fluid Mech. Vol. 560, pp. 103-122 2006.

3. Wu, C. C., Roberts, P. H. "Structure and stability of a spherical shock wave in a van der Waals gas" Q. J. Mech. Appl. Math. 49, No. 4, pp. 501-543, 1996.

4. Sharpe, J.A., "The Production of Elastic Waves by Explosion Pressures, I Theory and Empirical Field Observations," Geophysics 7, pp. 144-154 1942.

5. Blake, F. G. Jr., "Spherical Wave Propagation in Solid Media, "J. Accoust. Soc. Am. 24, pp 211-214, 1952.

6. Larson, D. B., "Spherical wave propagation in elastic media and its application to energy coupling for tamped and decoupled explosions," UCRL52655, 1979.

7. Moran, B. "Hydrodynamic test problems" Proceedings of the 5-Lab conference, UCRLCONF-212699, 2005.

8. Williams, T. O., Li, S., Brock, J. S., and Kamm, J. R. "Spherical Shell Analysis for Material Modeling” Los Alamos report LA-UR-05-8038, 2005.

9. Duvall, G.E., "Concepts of shock wave propagation" Bulletin of the Seismological Society of America, 52, No. 4, pp. 869-893, October 1962.

10. Tipton, R. E., Steinberg, D. J., and Tomita,Y., "Bubble Expansion and Collapse Near a Rigid Wall," Lawrence Livermore National Laboratory report UCRL-102945, 1990. 